\title{
Comparison of a dorsal closing wedge calcaneal osteotomy versus a posterosuperior prominence resection for the treatment of Haglund syndrome
}

\section{Zilu Ge}

Army Medical University

Lin Ma

Army Medical University

Hong Tang

Army Medical University

Mingyu Yang

Army Medical University

Aining Yang

Army Medical University

Chengsong Yuan

Army Medical University

Xu Tao

Army Medical University

Binghua Zhou

Army Medical University

Kanglai Tang

Army Medical University

Wan Chen ( $\nabla$ chenwanfred@foxmail.com )

Army Medical University https://orcid.org/0000-0001-5734-3432

Research article

Keywords: Haglund syndrome, dorsal closing wedge calcaneal osteotomy, posterosuperior prominence resection

Posted Date: January 21st, 2020

DOI: https://doi.org/10.21203/rs.2.21440/v1

License: (c) (1) This work is licensed under a Creative Commons Attribution 4.0 International License.

Read Full License 


\section{Abstract}

\section{Background}

Haglund syndrome is a common disease that causes posterior heel pain. The objective of this study was to compare the clinical outcomes between the dorsal closing wedge calcaneal osteotomy (DCWCO) and the posterosuperior prominence resection (PPR) for the treatment of this disease.

\section{Methods}

The retrospective study included 12 patients who received DCWCO and 32 who patients received PPR from January 2010 to August 2016. The patients were evaluated in terms of the American Orthopedic Foot Ankle Society ankle-hindfoot scale (AOFAS), the Victorian Institute of Sport Assessment Scale for Achilles tendinopathy (VISA-A), the Fowler-Philip angle, Bohler's angle, and the calcaneal pitch angle, both pre- and postoperatively (at 3 months, 6 months, 1 year, and the latest follow-up).

\section{Results}

Both groups showed a significance increase in their AOFAS and VISA-A scores after the operation. The DCWCO group showed a lower AOFAS score than the PPR group after 6 months ( $77.6 \pm 5.1$ vs. $82.8 \pm 7.8$, $P=0.037)$, but received higher scores in the latest follow-up $(98.2 \pm 2.3$, vs. $93.4 \pm 6.1, P=0.030)$. For the VISA-A scores, the DCWCO group had lower scores for 3 months $(56.9 \pm 13.9$, vs. $65.2 \pm 11.0, P=0.044)$, but showed higher scores in the latest follow-up $(98.2 \pm 2.6$, vs. $94.3 \pm 5.0, P=0.010)$ when compared with the PPR group. Both groups had a significant change in the Fowler-Philip angle and Bohler's angle after the operation. The postoperative Fowler-Philip angle in the DCWCO group was greater than that of the PPR group $\left(35.9^{\circ} \pm 4.9^{\circ}\right.$, vs. $\left.31.4^{\circ} \pm 6.2^{\circ}, P=0.026\right)$. However, there was no statistical difference in any other angle between the two groups, postoperatively.

\section{Conclusions}

The DCWCO group had poorer short-term clinical outcomes but better long-term function and symptom remission, as compared with the PPR group, in the treatment of Haglund syndrome.

\section{Introduction}

Haglund deformity, first described by Patrick Haglund in 1928, is a prominence in the posterolateral heel that causes posterior heel pain [1]. It is generally associated with insertional Achilles tendinopathy (IAT) and retrocalcaneal bursitis, comprising the concept of the Haglund triad or Haglund syndrome [2]. Surgery intervention is a suitable option for patients for who conservative treatment fails for more than 6 months [3]. Common surgical methods, including prominence resection, retrocalcaneal decompression, and endoscopic treatment, have been reported to have generally positive short-term clinical outcomes [4, 5]. However, few patients still suffer a different degree of pain after surgery treatment as mentioned 
above, especially after a posterosuperior prominence resection (PPR), requiring a surgical approach to contribute to better outcomes.

The dorsal closing wedge calcaneal osteotomy (DCWCO) was first described by Zadek for the treatment of IAT [6]. Keck and Kelly proved DCWCO as effective for eighteen patients in the treatment of Haglund's deformity [7]. Miller reported that wedge osteotomy of the calcaneus body combined with resection of the superior calcaneus in 16 patients (18 feet) showed good results [8]. Dimitrios treated IAT with dorsal wedge calcaneal osteotomy in 52 athletes, who showed great improvement in both function and pain relief [9]. Though this surgical technique approved effective in several studies, the comparison of the DCWCO versus the PPR in treatment of Haglund's syndrome has not been previously reported.

The purpose of this retrospective review was to evaluate the clinical outcomes between the DCWCO and the PPR. The ultimate outcomes focused on comparing the pain relief, ankle and Achilles tendon function improvement, anatomy change, and complications. Based on these comparisons, we aim to figure out an effective operation method for the treatment of Haglund's syndrome.

\section{Patients And Methods}

Records were reviewed for 50 patients with Haglund's syndrome who underwent a DCWCO or PPR from January 2009 to October 2014 at the Department of Sports Medicine, Southwest Hospital, the Army Medical University (Chongqing, China). A minimum of a 4-year follow-up after surgery was required for all patients.

Specific inclusion and exclusion criteria were designed for selecting patients. The inclusion criteria included an age older than 18 and failure in conservative treatment for more than 6 months. Exclusion criteria included Haglund's deformity with Achilles tendon rupture repairing, diabetes mellitus with or without neuropathic joint destruction, and local infection. Patients with no preoperative or 1-year followup radiographs were excluded. Six patients were excluded according to the criteria: Haglund's deformity with Achilles tendon rupture repairing: 3 ; diabetes: 1 , and incomplete radiographs: 2 . Forty-four patients were included in this series all of whom were divided into a DCWCO group $(n=12)$ and a PPR group $(n=$ 32). The following data were collected, including age, gender, body mass index (BMI), operative side, and follow-up duration.

\section{Operative Technique}

Dorsal closing wedge calcaneal osteotomy (DCWCO)

The surgical procedures were performed through a full-thickness lateral approach with patients in the lateral position and with regional spinal or epidural anesthesia administration. (Fig. 1A). A thigh tourniquet with $300 \mathrm{mmHg}$ pressure was used. When the calcaneus was exposed, the calcaneal body received a closing wedge osteotomy. The posterior cut was made from a point close to the base of the 
posterosuperior calcaneal tubercles to the point anterior to the weight-bearing aspect of the plantar calcaneal tubercle. The anterior osteotomy was performed at $90^{\circ}$ to the calcaneal underside surface. (Figure. 1B). The templates that contained angle, orientation, and width of the wedge to be removed were designed according to the preoperative lateral radiograph taken before the surgery. After a sagittal saw with a shorter blade completed the osteotomy, the calcaneus was fixed with partially threaded cannulated screws under the guidance of two Kirschner wires (Figure. 1C). The final fixation was confirmed by a Carm device. Then, after a closed negative pressure drainage was placed, the subcutaneous tissue was repaired with a $3-0$ absorbable suture, and the skin was closed with a $3-0$ nonabsorbable suture. (Fig. 2A and 2B)

\section{Posterosuperior prominence resection}

A similar incision and the same position of patients was used in the PPR surgery. The surgical procedures were performed as follows. After a full-thickness incision was created, the Haglund deformity was clearly exposed. The osteotomy was performed according to the preoperative evaluation. The prominence was completely removed by the saw, distally to proximally. The Achilles tendon was protected by retractors during the entire procedure. After smoothening the osteotomy surface, a Maximal dorsiflexing ankle was performed to confirm that there was no obvious impingement occurrence between the Achilles tendon and the calcaneal surface. A C-arm X-ray view showed that the superior calcaneal prominence was removed. After irrigating the wound, closed negative pressure drainage was used. The subcutaneous tissue was repaired with a $3-0$ absorbable suture, and the skin was closed with a $3-0$ nonabsorbable suture. (Fig. 2C and 2D)

\section{Postoperative Management}

For both groups, the negative pressure drainage was opened 6 hours after surgery, and was removed when blood present in the drainage was less than $120 \mathrm{ml}$. The skin sutures were removed 2 weeks postsurgery. For the DCWCO group, patients were kept as non-weightbearing and mainly did passive motions of the ankle during the initial 4 weeks. At 6 weeks to 3 months, partial weightbearing with the protection of a cast and stick was allowed. After 3 months, patients were allowed to walk with full weightbearing. For the B group, patients were suggested to avoid weightbearing in the first 3 weeks; however, full range of active motion exercise was allowed. After 3 weeks, patients could gradually walk with the stick. Walking with full weightbearing began at 6 weeks, postoperatively.

\section{Clinical And Radiographical Evaluation}

Functional evaluation, pain assessment, and anatomy change were included in the evaluation. The functional evaluation pre- and postoperatively (3months, 6 months, 1 year and latest follow-up) was included in the AOFAS score [10] and the VISA-A scoring system [11]. The anatomy change included the Fowler-Philip angle [12], Bohler's angle [13] and the calcaneal pitch angle [4] (Fig. 3). To avoid examiner 
bias, the clinical evaluation and data analysis were performed by two independent doctors who were blinded to the study.

\section{Statistical analysis}

All data were analyzed statistically using SPSS, version 22.0 (IBM, Chicago, IL). A Student t-test and a Mann-Whitney $\mathrm{U}$ test were used to compare the continuous variables. A Fisher's exact test was used to compare all categorical data. A Paired-Samples t-test was used to compare the pre- and postoperative radiological change in the same group. Statistical significance was accepted as $P<0.05$.

\section{Results}

The series included a total of 44 cases; 12 cases were in DCWCO group and the other 32 cases were in the PPR group. Patients demographics, including age, gender, body mass index, operative side, and follow-up time, were summarized in Table 1. There was no significant difference between the two groups.

All the functional scores pre- and postoperatively in the 3-month, 6-month, and 1-year periods, and the latest follow-up for both groups, were summarized in Table 2. The mean AOFAS and VISA-A scores showed no significant difference between the two groups before the operations. During the average of the 86.5 months of follow-up, the AOFAS score in the DCWCO group increased from $52.0 \pm 5.3$, preoperatively, to $98.2 \pm 2.3$ at the latest follow-up visit. While the mean AOFAS score in the PPR group increased from $50.7 \pm 5.1$ to $93.4 \pm 6.1$ at the latest visit, the average was 71.8 months of follow-up. When comparing the AOFAS score between the two groups after the 3 month and 6-month periods, the DCWCO scored lower than the PPR groups with significant alternation. However, the latest follow-up scores in the DCWCO group were significantly better than the PPR group. A similar trend was also seen in the outcomes of the VISA-A scale. The only difference was the outcomes after the 6-month period. The PPR groups scored $84.6 \pm 7.9$, better than the DCWCO group, whose score was $77.5 \pm 11.9$; however, there was no statistical difference.

The radiologic index pre- and postoperatively for both groups, including the Fowler-Philip angle, Bohler's angle, and the calcaneal pitch angle, were summarized in Table 3. The Fowler-Philip angle significantly decreased and Bohler's angle significantly increased after the operation in both groups; the calcaneal pitch angle was not changed.

Compared with the PPR group, the postoperative Fowler-Philip angles were significantly larger in the DCWCO groups. Meanwhile, all the other angles in the DCWCO groups had no statistical difference when compared with the PPR groups, pre- and postoperatively.

Postoperative complications developed in one patient in the DCWCO group and one patient in the PPR group. One patient in the DCWCO group had a delayed union of the calcaneal until four months after the operation; however, a year later, after the screws were removed, his pain was completely relieved. One 
patent in the PPR group had an incision infection; after treatment with antibiotics, the incision healed well, and no further debridement was performed.

Table 1

Summary of patient demographics

\begin{tabular}{|c|c|c|c|}
\hline Demographic variable & $\begin{array}{l}\text { DCWCO group } \\
(N=12)\end{array}$ & $\begin{array}{l}\text { PPR group } \\
(\mathrm{N}=12)\end{array}$ & $P$ value \\
\hline $\operatorname{Age}^{\Phi}$ & & & 0.380 \\
\hline Mean \pm SD & $32.8 \pm 9.7$ & $36.5 \pm 14.1$ & \\
\hline Median & 36.5 & 36.5 & \\
\hline Range (minimum -maximum) & $18-44$ & $18-63$ & \\
\hline Gender $\Psi$ & & & 0.647 \\
\hline Female, n (\%) & $3(25.0)$ & $6(23.1)$ & \\
\hline Male, n (\%) & $9(75.0)$ & $26(76.9)$ & \\
\hline $\mathrm{BMI}^{\Phi}$ & & & 0.399 \\
\hline Mean \pm SD & $24.4 \pm 2.8$ & $23.5 \pm 3.1$ & \\
\hline Median & 24.1 & 23.8 & \\
\hline Range (minimum -maximum) & $19.03-28.73$ & $18.37-31.88$ & \\
\hline Operative side ${ }^{\psi}$ & & & 0.504 \\
\hline Right & $5(41.7)$ & 18(56.3) & \\
\hline Left & $7(58.3)$ & 14(43.7) & \\
\hline Follow-up duration (months) ${ }^{\Phi}$ & & & 0.055 \\
\hline Mean \pm SD & $86.5 \pm 17.1$ & $71.8 \pm 22.4$ & \\
\hline Median & 81.5 & 76.5 & \\
\hline Range (minimum -maximum) & $65-116$ & $40-120$ & \\
\hline
\end{tabular}


Table 2

Comparison of functional scores pre- and postoperatively for both groups

\begin{tabular}{|c|c|c|c|}
\hline Scale & $\begin{array}{l}\text { DCWCO group } \\
(N=12)\end{array}$ & $\begin{array}{l}\text { PPR group } \\
(\mathrm{N}=32)\end{array}$ & $P$ value \\
\hline \multicolumn{4}{|l|}{ AOFAS score } \\
\hline Preoperatively ${ }^{\Phi}$ & $52.0 \pm 5.3$ & $50.7 \pm 5.1$ & 0.464 \\
\hline 3 months $^{\Phi}$ & $68.8 \pm 7.1$ & $75.7 \pm 7.3$ & $0.007^{*}$ \\
\hline 6 months $^{\Phi}$ & $77.6 \pm 5.1$ & $82.8 \pm 7.8$ & $0.037^{*}$ \\
\hline 1 years $^{\Phi}$ & $88.0 \pm 6.9$ & $89.4 \pm 8.6$ & 0.607 \\
\hline Latest follow-up $\Omega$ & $98.2 \pm 2.3$ & $93.4 \pm 6.1$ & $0.030^{*}$ \\
\hline \multicolumn{4}{|l|}{ VISA-A } \\
\hline Preoperatively ${ }^{\Phi}$ & $37.1 \pm 5.7$ & $35.7 \pm 7.1$ & 0.530 \\
\hline 3 months ${ }^{\Phi}$ & $56.9 \pm 13.9$ & $65.2 \pm 11.0$ & $0.044^{*}$ \\
\hline 6 months $\Omega$ & $77.5 \pm 11.9$ & $84.6 \pm 7.9$ & 0.118 \\
\hline 1 years $^{\Phi}$ & $90.6 \pm 8.0$ & $92.4 \pm 6.0$ & 0.427 \\
\hline Latest follow-up $\Omega$ & $98.2 \pm 2.6$ & $94.3 \pm 5.0$ & $0.010^{*}$ \\
\hline
\end{tabular}


Table 3

Comparison of the radiologic index pre- and postoperatively for both groups

\begin{tabular}{|c|c|c|c|}
\hline Measure & $\begin{array}{l}\text { DCWCO group } \\
(N=12)\end{array}$ & $\begin{array}{l}\text { PPR group } \\
(\mathrm{N}=32)\end{array}$ & $P$ value \\
\hline \multicolumn{4}{|l|}{ Fowler-Philip angle } \\
\hline Preoperatively ${ }^{\Phi}$ & $54.0^{\circ} \pm 5.2^{\circ}$ & $53.0^{\circ} \pm 5.6^{\circ}$ & 0.628 \\
\hline Postoperatively ${ }^{\Phi}$ & $35.9^{\circ} \pm 4.9^{\circ}$ & $31.4^{\circ} \pm 6.2^{\circ}$ & $0.026^{*}$ \\
\hline \multicolumn{4}{|l|}{ Bohler's angle } \\
\hline Preoperatively ${ }^{\Phi}$ & $32.1^{\circ} \pm 3.3^{\circ}$ & $32.0^{\circ} \pm 3.4^{\circ}$ & 0.994 \\
\hline Postoperatively $\Omega$ & $43.6^{\circ} \pm 2.8^{\circ}$ & $44.0^{\circ} \pm 4.7^{\circ}$ & 0.726 \\
\hline \multicolumn{4}{|c|}{ Calcaneal pitch angle } \\
\hline Preoperatively ${ }^{\Phi}$ & $25.5^{\circ} \pm 1.9^{\circ}$ & $25.1^{\circ} \pm 3.3^{\circ}$ & 0.704 \\
\hline Postoperatively ${ }^{\Phi}$ & $25.4^{\circ} \pm 1.8^{\circ}$ & $24.9^{\circ} \pm 2.4^{\circ}$ & 0.587 \\
\hline
\end{tabular}

\section{Discussion}

The most important finding of this study was that the DCWCO group had poorer short-term clinical outcomes but better long-term function and symptom remission as compared with the PPR group in the treatment of Haglund syndrome. Both operation methods decreased the Fowler-Philip angle and showed no statistical difference in other angles when compared with each other.

According to the results, both groups had a significant change in pain relief and function improvement and few surgical complications occurred. The PPR group had better outcomes than the DCWCO group in the short-term follow-up. Patients in the PPR group were able to get rid of most of the pain once surgery was performed, and could have a quick return to previous sports activity. The DCWCO group, by contrast, had a longer period of recovery due to more severe trauma caused by surgery. However, most of patients in this group found pain relief after the 1-year visit. The majority of the patients had a complete recovery and were able to take up exercise without any concern, as seen in the last follow-up. In general, the DCWCO group had poorer short-term symptoms but better long-term clinical outcomes as compared with the PPR group.

Haglund deformity is the abnormality of the posterosuperior part of the calcaneus. As it is near the insertion of Achilles tendon, the adjoining tendon and soft tissue are under irritation and pressed during ankle motion [14]. This impingement is considered the one of the primary causes of Achilles 
tendinopathy $[3,15]$. The surgical treatment for removing the impingement is required when patients fail in altering heel height, physiotherapy, local steroid injection, and other conservative treatments. The PPR can effectively eliminate the impingement between the Haglund deformity and the Achilles tendon. However, the impingement between the insertion of Achilles tendon and the calcaneal persisted, no matter how deep a resection was performed. Therefore, changing the structure of the calcaneus may be another option. The Fowler-Philip angle was reduced through the closing wedge calcaneal osteotomy. Meanwhile, the direction of the prominence was also altered. Therefore, there is no need for an extra resection of the Haglund deformity during the closing wedge calcaneal osteotomy. Both areas of impingement were avoided during the motion of the Achilles tendon. In addition, the insertion of the Achilles tendon was slightly elevated due to the osteotomy; the orientation of the tendon at the insertion was also changed. These biomechanical alternations may reduce the tendon stress [16]. All of the changes can eliminate the risk factors and moderate the progress of Achilles tendinopathy. However, an effective and rational biomechanical design is required to manifest the effects of anatomy change on tendon insertion in the future.

Besides, the different manage to the soft tissue near the tendon could also explain the results. Compared with DCWCO, the PPR injured the near soft tissue inevitable. The Kager's fat pads were believed to have associate with Achilles tendinopathy [17]. The size of fat pad differs between individuals with and without tendinopathy [18]. Cytokines produced by Hoffa's fat pad play a vital role in knee osteoarthritis [19]. The potential role of fat pad that mediate angiogenesis may aggravate the progression of Achilles tendinopathy [20]. Therefore, the PPR may affect the healing of Achilles tendinopathy by injuring the fat pad near the tendon, contributing to a worsen long-term clinical outcomes.

Different osteotomy methods and tips for the DCWCO have been reported previously. Boffeli set the anterior osteotomy at $90^{\circ}$ to the weight-bearing surface at the plantar apex; while the posterior cut was made from the calcaneal tubercles to the point posterior to the weight-bearing aspect of the plantar calcaneal tubercle [21]. It had a slight impact on the insertion of the plantar fascia and the anatomic shape of plantar tubercles. However, more attention should be concerned with protecting the Achilles tendon during this surgery. In addition, less bone fixation required a more discreet operation. Georgiannos performed a similar osteotomy as we did; they used K-wire to persevere the plantar bone-hinge [22] and showed good clinical outcomes in 64 athletes' feet [9]. Both methods provided are options to perform this surgery with less complications and more reproducibility.

In this study, when we reviewed all the patients who received Haglund's syndrome surgery, the DCWCO group had no patients who received an insertional Achilles tendon rupture repaired with an anchor, due to the limitation of the incision. In addition, the outcomes of patients who received a deformity resection and an anchor reconstruction were generally worse than those who received only a deformity resection. We believed that the worse clinical symptoms showed were induced by the ruptured insertional tendon. Therefore, we led patients who received a tendon rupture repairing into exclusion to avoid bias and interference. Though the incision we chose for the DCWCO was not suitable for repairing the tendon with an anchor at the same time, we believed that the DCWCO could also accompany tendon repairing to treat 
a Haglund's deformity with a tendon rupture. However, a more suitable incision and surgery technique was required.

However, there still are some limitations of the DCWCO. First, the insertion of the Achilles tendon would get mildly raised due to the osteotomy. The effect of an anatomy change has not been proven in biomechanical testing. Although the long-term follow-up did not show the symptom in patients, we cannot ignore it. The good clinical outcomes can by interpreted by the possibility that the tendon adapts to the structure change and tendon stress is released. However, it can also be explained by the small sample size and required longer follow-up. Therefore, an effective and rational biomechanical design is required in the future. Second, although the calcaneal union did not appear in our patients, it is still a potential surgery complication; and it remains a challenge for the surgeon who performs this surgery for the first time. In addition, early function rehabilitation was required due to the longer recovery period; and the second surgery for removing the two screws bring additional costs and pain for patients.

\section{Conclusion}

This study involved a long-term follow-up comparing the DCWCO with the PPR in the treatment of Haglund syndrome. The DCWCO was found to have poorer short-term clinical outcomes, but better functional improvement in the long-term follow-up as compared with the PPR technique.

\section{Abbreviations}

DCWCO:dorsal closing wedge calcaneal osteotomy; PPR:posterosuperior prominence resection; IAT:insertional Achilles tendinopathy; AOFAS:American Orthopedic Foot Ankle Society ankle-hindfoot scale; VISA-A:Victorian Institute of Sport Assessment Scale for Achilles tendinopathy.

\section{Declarations}

\section{Acknowledgements}

Not applicable.

\section{Authors' contributions}

All surgical procedures were carried out by Pro. Tang KL. Ge ZL and Ma L contributed in the statistical analysis and drafting of the manuscript. Tang $\mathrm{H}$ and Yang MY contributed in the radiographic assessment and data collecting. Yuan CS, Tao X and Zhou BH contributed in the literature searching and revision of the manuscript. Tang $\mathrm{KL}$ and Chen $\mathrm{W}$ contributed in the research design and revision of the manuscript. All authors read and approved the final manuscript.

\section{Funding}

Not applicable. 
Availability of data and materials

All data and materials regarding the study are available from the corresponding author.

\section{Ethics approval and consent to participate}

This study was approved by the Clinical Academic Committee of the Army Military Medical University Southwest Hospital and conducted in compliance with the Helsinki Declaration. Written consent was acquired for all patients.

\section{Consent for publication}

Not applicable.

\section{Competing interests}

The authors declare that they have no competing interests.

\section{Author details}

Department of Orthopeadics/Sports Medicine Center, State Key Laboratory of Trauma, Burn and Combined Injury, Southwest Hospital, Third Military Medical University (Army Medical University), Chongqing, China.

\section{References}

[1] Haglund P. Beitrag zur klinik der achillessehne. Zeitschrift Orthop 1928;49:49-58.

[2] DeVries JG, Summerhays B, Guehlstorf DW. Surgical correction of Haglund's triad using complete detachment and reattachment of the Achilles tendon. J Foot Ankle Surg 2009;48(4):447-51.

[3] Longo UG, Ronga M, Maffulli N. Achilles Tendinopathy. Sports Med Arthrosc Rev 2018;26(1):16-30.

[4] Ahn JH, Ahn CY, Byun CH, Kim YC. Operative Treatment of Haglund Syndrome With Central Achilles Tendon-Splitting Approach. J Foot Ankle Surg 2015;54(6):1053-6.

[5] Lui TH, Lo CY, Siu YC. Minimally Invasive and Endoscopic Treatment of Haglund Syndrome. Foot and ankle clinics 2019;24(3):515-31.

[6] Zadek I. An operation for the cure of achillobursitis. 1939;43(2):542-6.

[7] Keck SW, Kelly PJ. Bursitis Of the Posterior Part Of the Heel; Evaluation Of Surgical Treatment Of Eighteen Patients. J Bone Joint Surg Am 1965;47:267-73.

[8] Miller AE, Vogel TA. Haglund's deformity and the Keck and Kelly osteotomy: a retrospective analysis. J Foot Surg 1989;28(1):23-9. 
[9] Georgiannos D, Lampridis V, Vasiliadis A, Bisbinas I. Treatment of Insertional Achilles Pathology With Dorsal Wedge Calcaneal Osteotomy in Athletes. Foot Ankle Int 2017;38(4):381-7.

[10] Kitaoka HB, Alexander IJ, Adelaar RS, Nunley JA, Myerson MS, Sanders M. Clinical rating systems for the ankle-hindfoot, midfoot, hallux, and lesser toes. Foot Ankle Int 1994;15(7):349-53.

[11] Robinson JM, Cook JL, Purdam C, Visentini PJ, Ross J, Maffulli N, et al. The VISA-A questionnaire: a valid and reliable index of the clinical severity of Achilles tendinopathy. $\mathrm{Br} \mathrm{J}$ Sports Med 2001;35(5):335-41.

[12] Fowler A, Philip JF. Abnormality of the calcaneus as a cause of painful heel its diagnosis and operative treatment. Br J Surg 1945;32(128):494-8.

[13] Böhler L. Diagnosis, pathology, and treatment of fractures of the os calcis. JBJS 1931;13(1):75-89.

[14] Vaishya R, Agarwal AK, Azizi AT, Vijay V. Haglund's Syndrome: A Commonly Seen Mysterious Condition. Cureus 2016;8(10):e820.

[15] Bah I, Kwak ST, Chimenti RL, Richards MS, J PK, Samuel Flemister A, et al. Mechanical changes in the Achilles tendon due to insertional Achilles tendinopathy. Journal of the mechanical behavior of biomedical materials 2016;53:320-8.

[16] Perlman MD. Enlargement of the entire posterior aspect of the calcaneus: treatment with the Keck and Kelly calcaneal osteotomy. J Foot Surg 1992;31(5):424-33.

[17] Ward ER, Andersson G, Backman LJ, Gaida JE. Fat pads adjacent to tendinopathy: more than a coincidence? Br J Sports Med 2016;50(24):1491-2.

[18] Culvenor AG, Cook JL, Warden SJ, Crossley KM. Infrapatellar fat pad size, but not patellar alignment, is associated with patellar tendinopathy. Scand J Med Sci Sports 2011;21(6):e405-11.

[19] Pottie P, Presle N, Terlain B, Netter P, Mainard D, Berenbaum F. Obesity and osteoarthritis: more complex than predicted! Ann Rheum Dis 2006;65(11):1403-5.

[20] Nemschak G, Pretterklieber ML. The Patellar Arterial Supply via the Infrapatellar Fat Pad (of Hoffa): A Combined Anatomical and Angiographical Analysis. Anatomy research international 2012;2012:713838.

[21] Boffeli TJ, Peterson MC. The Keck and Kelly wedge calcaneal osteotomy for Haglund's deformity: a technique for reproducible results. J Foot Ankle Surg 2012;51(3):398-401.

[22] Georgiannos D, Kitridis D, Bisbinas I. Dorsal closing wedge calcaneal osteotomy for the treatment of Insertional Achilles Tendinopathy: A technical tip to optimize its results and reduce complications. Foot Ankle Surg 2018;24(2):115-8. 


\section{Figures}
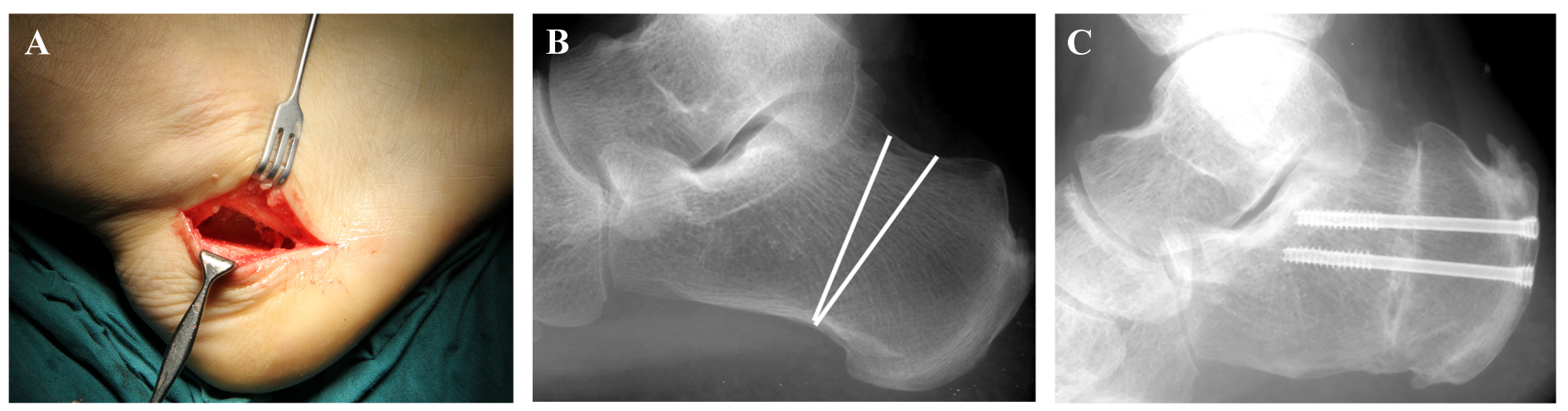

\section{Figure 1}

(A) the incision of the operation. (B) The cut line of the calcaneus. (C). Two partially threaded cannulated screws used for fixation.
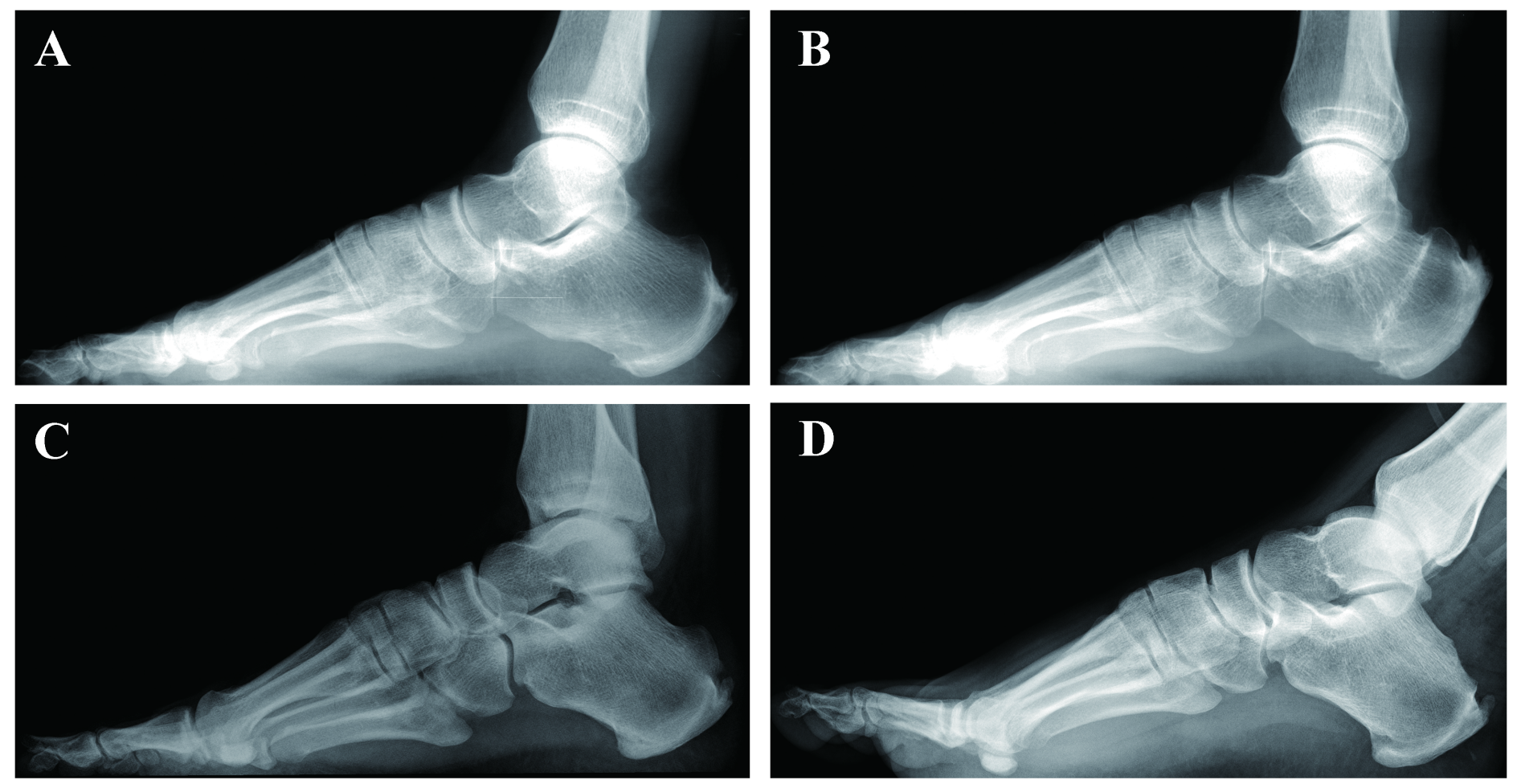

\section{Figure 2}

Pre- and postoperative manifestations of the dorsal closing wedge calcaneal osteotomy ( $\mathrm{A}$ and $\mathrm{B}$ ); Preand postoperative manifestations of the posterosuperior prominence resection (C and D). 


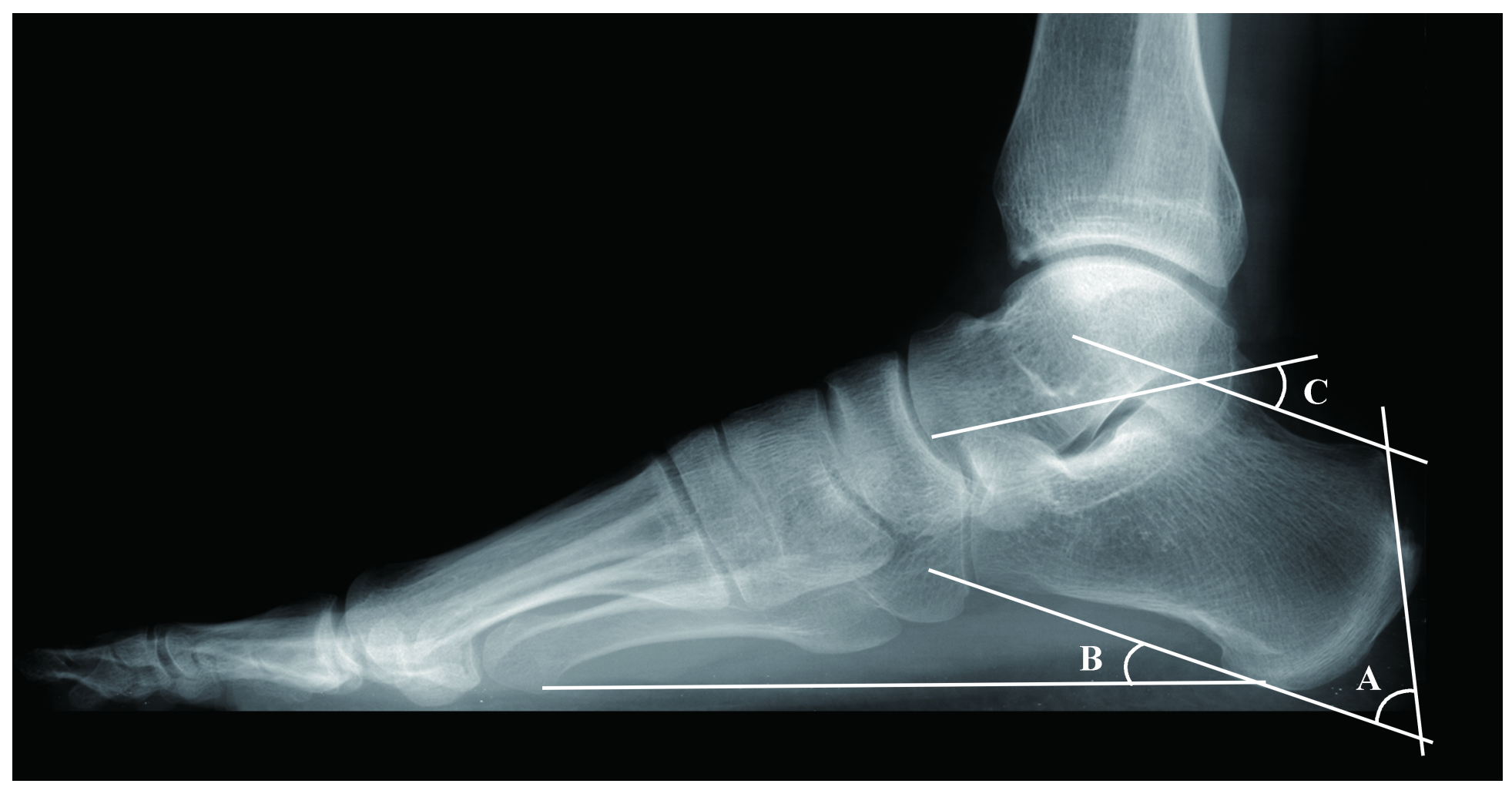

Figure 3

A Radiographic index on the standing lateral foot radiograph. A, The Fowler-Philip angle; B, The Calcaneal pitch angle; C, Bohler's angle. 